\title{
NLO QCD corrections to off-shell tīj production at the LHC
}

\author{
Małgorzata Worek* $\dagger$ \\ Institute for Theoretical Particle Physics and Cosmology \\ RWTH Aachen University, D-52056 Aachen, Germany \\ E-mail: worek@physik.rwth-aachen.de
}

\begin{abstract}
A short summary of results for top-quark pair production in association with a jet with NLO QCD off-shell effects at the LHC is given. The calculation is based on the matrix element for the $p p \rightarrow e^{+} v_{e} \mu^{-} \bar{v}_{\mu} b \bar{b} j$ process. All quantum effects are properly accounted for, which amounts to including all Feynman diagrams that result in this final state with interference effects as well as non-zero top-quark width effects. Results for total cross sections and a few differential distributions at the LHC Run1 energy of $\sqrt{s}=8 \mathrm{TeV}$ are presented. In addition, the size of top-quark off-shell effects is quantified for the total cross section in presence of rather inclusive cuts on all final states. Numerical results shown in this proceedings have been obtained with the HELAC-NLO Monte Carlo program.
\end{abstract}

Loops and Legs in Quantum Field Theory

24-29 April 2016

Leipzig, Germany

* Speaker.

${ }^{\dagger}$ TTK-16-26 


\section{Introduction}

Top-quark studies are currently driven by two Large Hadron Collider (LHC) experiments, i.e. ATLAS and CMS. Besides the determination of the top-quark mass, $m_{t}$, and the strong coupling constant, $\alpha_{s}$, key measurements include fiducial cross sections, various infra-red safe differential distributions as well as spin correlations and top-quark couplings to gauge bosons and the Standard Model (SM) Higgs boson. In addition, work on constraining parton distribution functions for the dominant production channel, i.e. the $g g$ channel is ongoing. The top-quark phenomenology provides a unique laboratory where our understanding of the strong interactions, both in the perturbative and non-perturbative regimes, can be tested. Searches for rare top-quark decays to probe physics beyond the SM also play a prominent role at the LHC. Finally, $t \bar{t}$ production is the dominant background process for studies of the SM Higgs boson and for new physics searches. The top-quark is an extremely short-lived resonance and only its decay products can be detected experimentally. In general, for comparison with data, theoretical predictions must include topquark decays. In the SM, the top-quark decays almost exclusively to a $W$-boson and a $b$-quark. The experimentally cleanest top-quark decay channel, which comprises leptonic decays of both $W$-gauge bosons, is called the di-lepton channel. The signature for this channel consists of two well-isolated and oppositely charged leptons with high transverse momentum, missing transverse momentum, $p_{T}^{\text {miss }}$, from invisible neutrinos and two jets that originate from bottom quarks. However, due to the large collision energy at the LHC, top-quark pairs are abundantly produced with large energies and high transverse momenta. Thus, the probability for additional radiation increases making the $t \bar{t} j$ final state measurable with high statistics. To estimate the size of the $t \bar{t} j$ contribution in the inclusive $t \bar{t}$ sample we show in Table 1 the cross section for the on-shell $p p \rightarrow t \bar{t} j$ production at NLO in QCD with various $p_{T}(j)$ cuts on the hard jet. Also shown is its ratio to the inclusive $p p \rightarrow t \bar{t}$ production at NNLO. Both results are given for the LHC Run2 energy of $13 \mathrm{TeV}$ and the top-quark mass of $m_{t}=173.2 \mathrm{GeV}$. In addition, for parton distribution functions (PDFs) CT14nlo and CT14nnlo sets have been employed [1]. NLO results have been obtained

\begin{tabular}{||c||cc||cc||}
\hline \hline$p_{T}(j)[\mathrm{GeV}]$ & \multicolumn{1}{|c||}{$\sigma_{t \bar{t} j}^{N L O}[\mathrm{pb}]$} & $\sigma_{t \bar{t} j}^{N L O} / \sigma_{t \bar{t}}^{N N L O}$ & {$[\%]$} \\
\hline \hline 40 & $296.97 \pm 0.29$ & 37 & \\
60 & $207.88 \pm 0.19$ & 26 & \\
80 & $152.89 \pm 0.13$ & 19 & \\
100 & $115.60 \pm 0.14$ & 14 & \\
120 & $89.05 \pm 0.10$ & 11 & \\
\hline \hline
\end{tabular}

Table 1: The NLO cross section for the on-shell $p p \rightarrow t \bar{t} j$ production with various $p_{T}(j)$ cut on the hard jet and its ratios to $p p \rightarrow t \bar{t}$ production at NNLO for the LHC Run 2 energy of $13 \mathrm{TeV}$.

with the HELAC-NLO package [2], while the NNLO result, $\sigma_{t \bar{t}}^{N N L O}=807 \mathrm{pb}$, has been calculated with the ToP++ program [3]. For $p_{T}(j)>40 \mathrm{GeV}$ almost $40 \%$ of the top anti-top events are actually accompanied by an additional hard jet. Therefore, for a better understanding of $p p \rightarrow t \bar{t}$ kinematics at the LHC it is essential to also study $p p \rightarrow t \bar{t} j$ production in more details. However, $p p \rightarrow t \bar{t} j$ production is important on its own. It can be employed in the top-quark mass 

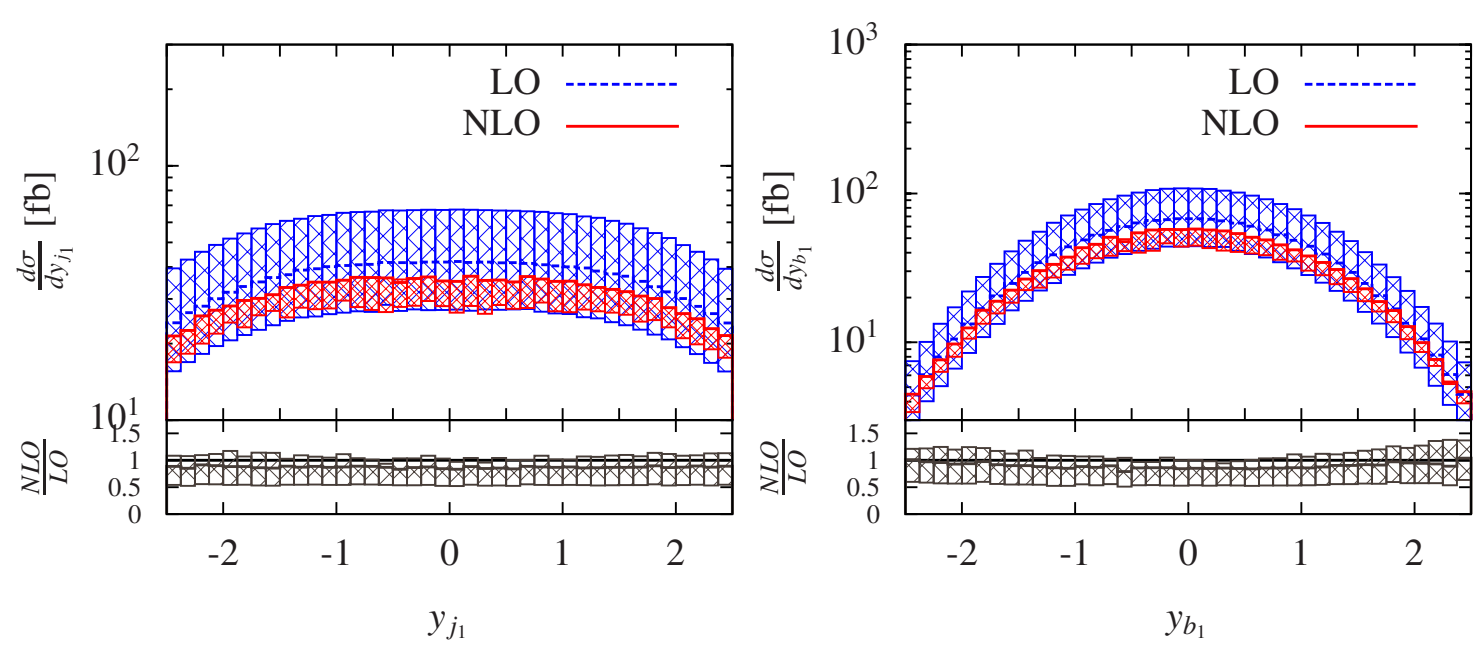

Figure 1: Differential cross section distributions as a function of rapidity of the hardest light- and b-jet for $p p \rightarrow e^{+} v_{e} \mu^{-} \bar{v}_{\mu} b \bar{b} j+X$ at the LHC with $\sqrt{s}=8 \mathrm{TeV}$. The uncertainty bands depict the scale variation. Lower panels display differential $K$ factors and their uncertainty bands.

extraction by studying normalised differential cross sections as a function of the (inverse) invariant mass of the $t \bar{t} j$ system [4]. The method has already been successfully used by experimental groups at the LHC [5,6]. Additionally, $t \bar{t} j$ constitutes an important background to the SM Higgs boson production in the vector boson fusion process, $p p \rightarrow H j j \rightarrow W^{+} W^{-} j j[7,8]$. Indeed, for the $2 \ell p_{T}^{\text {miss }}+$ jets final state not $p p \rightarrow t \bar{t}$ but the $p p \rightarrow t \bar{t} j$ production process is the dominant background process. To understand this better one needs to recall that the VBF signature consists, among other things, of two tagging jets, denoted as $j_{1}^{\text {tag }}$ and $j_{2}^{\text {tag }}$, that have to be very well separated in the rapidity plane. In practice, this means that both tagging jets have to fulfil a large rapidity cut $\Delta y\left(j_{1}^{\text {tag }} j_{2}^{\text {tag }}\right)=\left|y\left(j_{1}^{\text {tag }}\right)-y\left(j_{2}^{\text {tgg }}\right)\right|>4$ and they are required to reside in opposite hemispheres of the detector, i.e. $y\left(j_{1}^{\text {tag }}\right) \times y\left(j_{2}^{\text {tag }}\right)<0$. In case of $p p \rightarrow t \bar{t} \rightarrow W^{+} W^{-} b \bar{b} \rightarrow e^{+} v_{e} \mu^{-} \bar{v}_{\mu} b \bar{b}$ two tagging jets are $b$-jets. From Figure 1 we can see that they are centrally distributed in the rapidity plane. Thus, the above two conditions will dramatically decrease the contribution from this process. On the other hand, for $p p \rightarrow t \bar{t} j \rightarrow W^{+} W^{-} b \bar{b} j \rightarrow e^{+} v_{e} \mu^{-} \bar{v}_{\mu} b \bar{b} j$ production it is sufficient that only one of the two tagging jets is the $b$-jet since we also have a light-jet. The latter is distributed uniformly in the rapidity plane, as can be observed in Figure 1. Therefore, in that case a quite substantial part of the cross section remains causing the $t \bar{t} j$ production process to dominate among various background processes. Furthermore, $p p \rightarrow t \bar{t} j$ plays a very important role in searches for physics beyond the SM. For example, it is one of the main backgrounds to processes such as supersymmetric particle production [9]. Depending on the specific model, typical signals also include $2 \ell^{ \pm}+E_{T}^{\text {miss }}+$ jets, where $E_{T}^{\text {miss }}$ is due to the escaping lightest supersymmetric particle. Another exemple comprises the production of top-quark flavour violating states like for example $M \rightarrow \tilde{t} q$, where $\tilde{t}=t$ or $\bar{t}$. Such states can be singly produced in association with a top-quark at the LHC. Thus, the direct signature will consist of a $t j$ (or $\bar{t} j$ ) resonance in $t \bar{t} j$ events [10]. In view of a correct interpretation of the signals of new physics, which might be extracted from data, it is of great importance to understand the background process as precisely as possible. In this respect, 

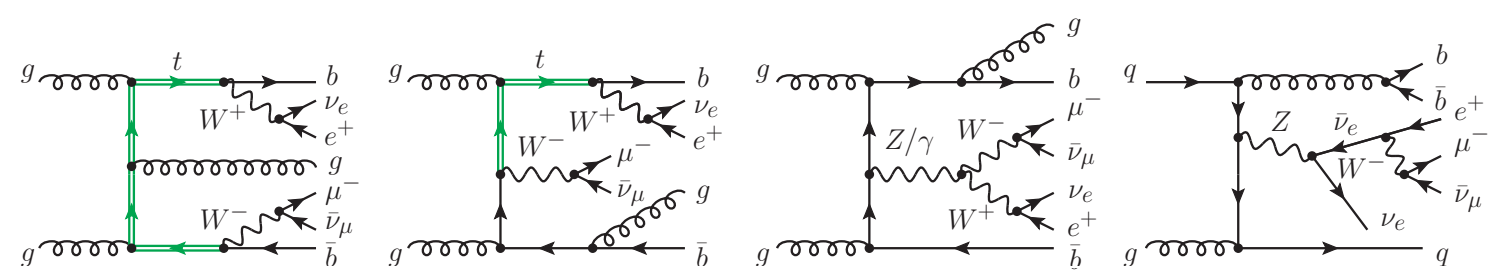

Figure 2: Representative Feynman diagrams, involving two (first diagram), one (second diagram) and no top-quark resonances (third diagram), contributing to the $p p \rightarrow e^{+} v_{e} \mu^{-} \bar{v}_{\mu} b \bar{b} j$ process at $\mathscr{O}\left(\alpha_{s}^{3} \alpha^{4}\right)$. The last diagram with a single $W$ boson resonance contributes to the off-shell effects of $W$ gauge bosons.

the need of precision theoretical predictions for $p p \rightarrow t \bar{t} j$ production process is indisputable. NLO QCD corrections for the $p p \rightarrow t \bar{t} j$ production process have been first calculated with on-shell topquarks $[11,12]$. In the next step decays in the so-called narrow-width-approximation (NWA) have been included, first at LO [13] and later on at NLO [14]. In this approximation various production and decay stages are treated sequentially. The NWA method allows to neglect single-resonant and non-resonant amplitude contributions as well as all interferences and off-shell effects of the top-quark. This of course leads to significant simplifications in calculations of higher order corrections for top-quark physics. Finally, complete NLO QCD corrections to $p p \rightarrow t \bar{t} j$ in the di-lepton channel have been recently provided [22]. Here the approximation that top quarks are only produced on-shell has been dropped, and the fully realistic final state $p p \rightarrow e^{+} v_{e} \mu^{-} \bar{v}_{\mu} b \bar{b} j$ has been considered. Resonant and non-resonant top quark contributions as well as all interference effects among them have been consistently taken into account. In addition, non-resonant and off-shell effects due to the finite $W$ gauge boson width were included. A few examples of Feynman diagrams contributing to the LO process at $\mathscr{O}\left(\alpha_{s}^{3} \alpha^{4}\right)$ are presented in Figure 2. Such calculations are extremely complex and the question remains whether they are really needed. Indeed, the size of the top quark off-shell effects is controlled by the ratio of the top-quark width to the top-quark mass $\Gamma_{t} / m_{t} \approx 10^{-2}$. Thus, the contributions that are neglected in the NWA should be suppressed at least for sufficiently inclusive observables like the total cross sections. Certainly, in case of $p p \rightarrow t \bar{t}$ and $p p \rightarrow t \bar{t} j$ productions as well as for $p p \rightarrow t \bar{t} H$, these effects have been found to be of the order of $1 \%-2 \%$ for the total cross section [15-22]. However, they can be strongly enhanced in exclusive observables that play an important role in studies of the Higgs boson and searches for new physics [23]. Before presenting a few results for $p p \rightarrow e^{+} v_{e} \mu^{-} \bar{v}_{\mu} b \bar{b} j$ production at $\mathscr{O}\left(\alpha_{s}^{4} \alpha^{4}\right)$ let us shortly mention here that results for $p p \rightarrow t \bar{t} j$ at NLO accuracy matched with parton showers are also available [24-26].

\section{Outline of the Calculation}

NLO QCD corrections to $p p \rightarrow e^{+} v_{e} \mu^{-} \bar{v}_{\mu} b \bar{b} j+X$ have been calculated with the HELACNLO Monte Carlo program, which has already been extensively used for top-quark related studies at NLO in QCD [27-32]. HelaC-NLO is an extension of the HelaC-PhEgas LO MC program [33,34], which is based on off-shell Dyson-Schwinger recursive equations for amplitudes calculation. The HELAC-1LOOP program [35], based on the Ossola-Papadopoulos-Pittau reduc- 
tion technique [36] and the reduction code CUTTOoLs [37], has been used to deal with virtual corrections. For the evaluation of the scalar integrals the ONELOOP library has been employed [38]. Singularities from soft or collinear parton emission have been isolated via subtraction methods for NLO QCD. Specifically, two methods have been made use of, the commonly used CataniSeymour dipole subtraction [39], and the Nagy-Soper subtraction scheme [40], both implemented in the HelaC-DiPOLES software [41]. Phase space integration has been executed with the help of KALEU [42]. The process under consideration requires a special treatment of unstable top-quarks. We regularize intermediate top-quark resonances in a gauge-invariant way via the complex-massscheme [43]. In this approach the top-quark width $\Gamma_{t}$ is incorporated into the definition of the (squared) top-quark mass through $\mu_{t}^{2}=m_{t}^{2}-i m_{t} \Gamma_{t}$. As a consequence top-quark contributions are consistently described by Breit-Wigner distributions at the cost of evaluating all matrix elements with complex top-quark masses.

\section{Numerical Results for the LHC}

In the following we present predictions for the total cross section and a few differential observables at the LHC for the collider energy of $8 \mathrm{TeV}$. The input parameters used in our calculations are given in Table 2. We employ the MSTW2008NLO (LO) PDFs [46] and use the running of

\begin{tabular}{||c||c||}
\hline \hline$G_{F}=1.16637 \cdot 10^{-5} \mathrm{GeV}^{-2}$ & $m_{t}=173.3 \mathrm{GeV}[44]$ \\
$m_{W}=80.399 \mathrm{GeV}$ & $\Gamma_{W}=2.09974 \mathrm{GeV}$ \\
$m_{Z}=91.1876 \mathrm{GeV}$ & $\Gamma_{\mathrm{Z}}=2.50966 \mathrm{GeV}$ \\
$\Gamma_{t}^{L O}=1.48132 \mathrm{GeV}[45]$ & $\Gamma_{t}^{N L O}=1.3542 \mathrm{GeV}[45]$ \\
\hline \hline
\end{tabular}

Table 2: $S M$ parameters in the $G_{\mu}$ scheme.

the strong coupling constant $\alpha_{s}$ with two-loop (one-loop) accuracy as provided by the LHAPDF library. Suppressed contributions induced by the bottom-quark parton density are neglected. All final-state partons with pseudorapidity $|\eta|<5$ are recombined into jets via the infrared safe anti- $k_{T}$ jet algorithm [47] where a cone size of $R=0.5$ has been chosen. Additional cuts are imposed on the transverse momenta and the rapidity of recombined jets as well as the distance between jets:

$$
p_{T}(j)>40 \mathrm{GeV}, \quad|y(j)|<2.5, \quad \Delta R_{j j}>0.5 .
$$

Basic selection is also applied to decay products of top-quarks:

$$
p_{T}(\ell)>30 \mathrm{GeV}, \quad p_{T}^{\text {miss }}>40 \mathrm{GeV}, \quad \Delta R_{\ell \ell}>0.4, \quad \Delta R_{\ell j}>0.4, \quad|y(\ell)|<2.5 .
$$

The factorisation and renormalisation scales, $\mu_{F}$ and $\mu_{R}$ respectively, have been set to a common value $\mu_{0}=m_{t}$. We start with a discussion of the total cross section. At the central scale, we have obtained:

$$
\begin{aligned}
& \sigma_{p p \rightarrow e^{+} v_{e} \mu^{-} \bar{v}_{\mu} b \bar{b} j}^{L O}=183.1_{-64.2(35 \%)}^{+112.2(61 \%)} \mathrm{fb}, \\
& \sigma_{p p \rightarrow e^{+} v_{e} \mu^{-} \bar{v}_{\mu} b \bar{b} j}^{N L O}=159.7_{-7.9(5 \%)}^{-33.1(21 \%)} \mathrm{fb} .
\end{aligned}
$$


Thus, the $\mathscr{K}$ factor is 0.87 . Additionally, the inclusion of the NLO corrections reduces the scale dependence from $61 \%$ to $21 \%$. In the next step we present various differential distributions obtained by applying the cuts specified in Eq. (3.1) and Eq. (3.2). In Figure 3 differential cross section distributions as function of the transverse momentum of the hardest (in $p_{T}$ ) light- and $b$-jet are shown. The upper panels display absolute LO (the blue-dashed curve) and NLO (the red-solid curve) predictions together with corresponding uncertainty bands, which are calculated as maximum and minimum out of the following set $\left\{0.5 m_{t}, m_{t}, 2 m_{t}\right\}$. The lower panels display the differential $\mathscr{K}$ factor. Higher order corrections do not simply rescale the shape of the LO distributions. Instead distortions of the order of $-50 \%$ are visible within the plotted range. Negative NLO corrections in $p_{T}$ tails simply mean that the LO result is higher than the NLO one. Moreover, we note that the NLO error bands do not fit within the LO ones as one should expect from a well-behaved perturbative expansion. This situation can be cured with a judicious choice of a dynamic scale. The dynamic scale should take into account $p_{T}$ of top-quark decay products so its value would increase in the tails of $p_{T}$ distributions. On the other hand, close to the $t \bar{t}$ treshold, it should reduce to the top-quark mass since there $\mu_{0}=m_{t}$ behaves correctly. On the contrary, for dimensionless distributions moderate and quite stable NLO corrections are obtained. As an example in Figure 1 differential cross section distributions as a function of the rapidity of the hardest light- and $b$-jet are given, whereas in Figure 3 differential cross section distributions as a function of $\Delta R_{b_{1} b_{2}}=\sqrt{\Delta \phi_{b_{1} b_{2}}^{2}+\Delta y_{b_{1} b_{2}}^{2}}$ and $R_{e^{+} \mu^{-}}=\sqrt{\Delta \phi_{e^{+} \mu^{-}}^{2}+\Delta y_{e^{+} \mu^{-}}^{2}}$ are provided. These angular distributions receive contributions from all scales, most notably from those that are sensitive to the threshold for the $t \bar{t} j$ production. For our scale choice, effects of the phase space regions close to this threshold dominate and a dynamic scale will not alter this behaviour. And finally, in Figure 3 the differential cross section distribution as a function of the invariant mass of the positron and the $b$-jet, $M_{b e^{+}}$, is shown. Also given there is the differential cross section distribution as a function of the invariant mass of the top-quark in the vicinity of its resonance, $M_{t}$. Both observables are particularly sensitive to off-shell top-quark effects. We will concentrate first on the $M_{b e^{+}}$distribution, where the $e^{+} b$ pair is formed by selecting the $b$-jet that yields the smallest invariant mass. In the LO NWA this observable is characterized by a sharp upper bound

$$
M_{b e^{+}}=\sqrt{m_{t}^{2}-m_{W}^{2}} \approx 153 \mathrm{GeV} .
$$

Additional radiation and off-shell top-quark effects as well as off-shell effects of $W$ gauge bosons introduce a smearing to the region, which is highly sensitive to the details of the description of the process. In the case of $t \bar{t}$ production, this observable has proved to be particularly important for the extraction of the top-quark mass with a very high precision $[48,49]$. The top quark mass can be determined either from the shape of the distribution away from the kinematical endpoint or from the behavior of the observable in the vicinity of that point. In the region below $153 \mathrm{GeV}$, the NLO corrections to $M_{b e^{+}}$are moderate, however, above the kinematic upper bound they can be as high as $100 \%$. Even though the contribution to the total cross section from this region is fairly small, the impact on the top-mass measurement might be non-negligible. Higher order corrections and off-shell contributions impact greatly another observable that is highly sensitive to the top-quark mass extraction, i.e. the invariant mass of the top quark, $M_{t}=M_{b \ell v_{\ell}}$. In the presence of off-shell top-quark contributions, a clear Breit-Wigner structure is visible. We can see that below the top-quark resonance the size of NLO corrections is huge due to final-state gluon radiation that 

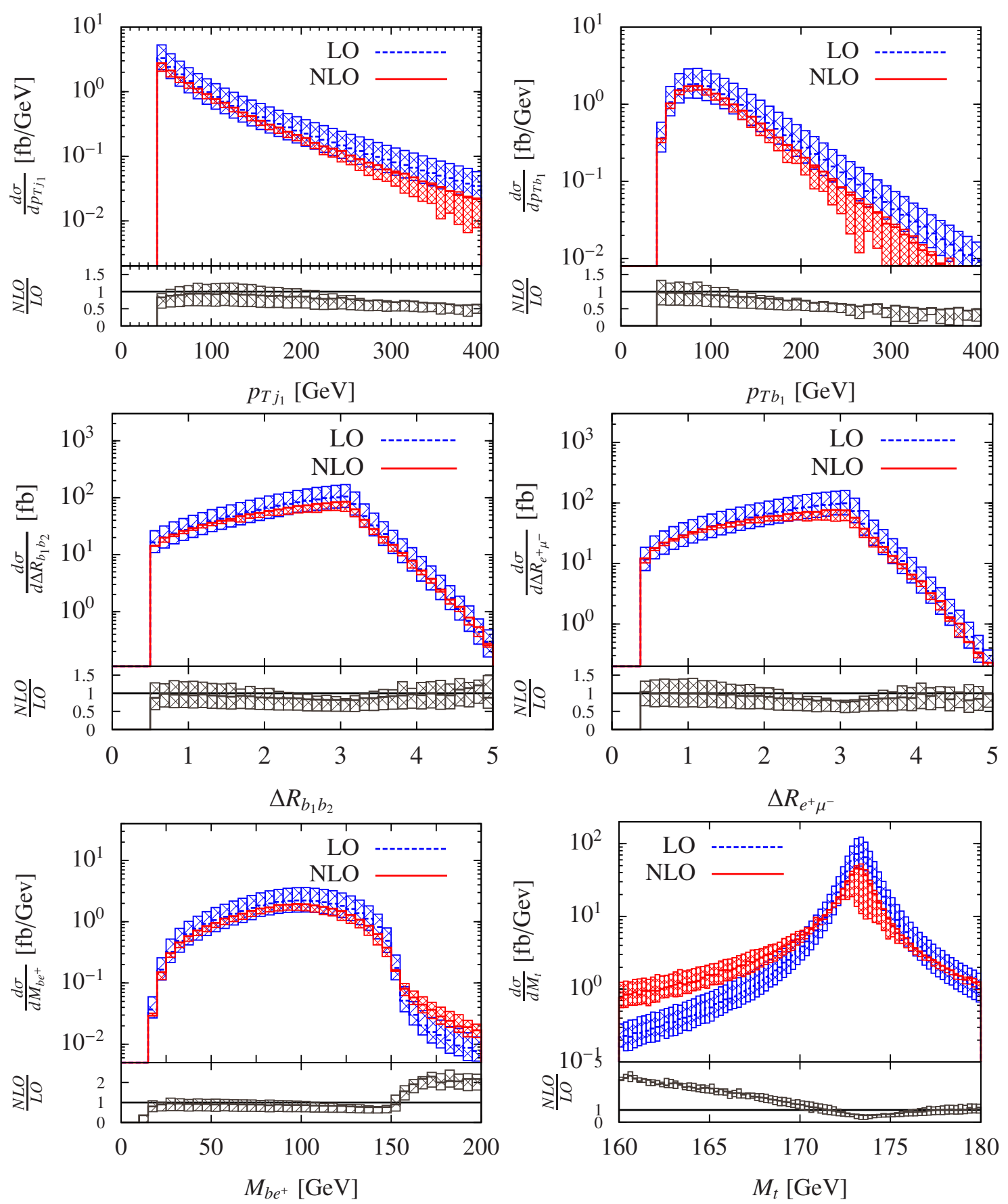

Figure 3: Differential cross section distributions as a function of $p_{T}\left(j_{1}\right), p_{T}\left(b_{1}\right), \Delta R_{b_{1} b_{2}}, \Delta R_{e^{+} \mu^{-}}, M_{b e^{+}}$, and $M_{t}$ for $p p \rightarrow e^{+} v_{e} \mu^{-} \bar{v}_{\mu} b \bar{b} j+X$ at the LHC with $\sqrt{s}=8 \mathrm{TeV}$. The uncertainty bands depict the scale variation. Lower panels display differential $K$ factors and their uncertainty bands.

is not recombined with the top-quark decay products. Once the kinematical region above $M_{t} \sim m_{t}$ is reached NLO corrections become moderate. Since top quark mass measurements are carried out using the $M_{t}$ distribution precise theoretical modeling of top-quark decays is of paramount impor- 
tance. When aiming at the $m_{t}$ extraction with a precision of $1 \mathrm{GeV}$ or less, theoretical predictions that are used in such analyses should go beyond simple approximation of factorising top-quark production and decays.

In summary, NLO QCD corrections to the $p p \rightarrow e^{+} v_{e} \mu^{-} \bar{v}_{\mu} b \bar{b} j+X$ production process have been calculated. We have shown that they are moderate for the total cross section, i.e. of the order of $13 \%$. Nevertheless, their impact on some differential distributions is much larger. A special case are differential observables that are used in the top-quark mass extraction. Shape-based $m_{t}$ measurements depend on precise modeling of differential distributions, thus, top-quark decays in NWA are simply not good enough.

\section{Acknowledgement}

The author would like to thank the organizers of the $13^{\text {th }}$ Workshop on Loops and Legs in Quantum Field Theory for a kind invitation and very pleasant atmosphere during the conference. The work was supported by the DFG under Grant: "Signals and Backgrounds Beyond Leading Order. Phenomenological studies for the LHC".

\section{References}

[1] S. Dulat et al., Phys. Rev. D 93 (2016) no.3, 033006.

[2] G. Bevilacqua et al., Comput. Phys. Commun. 184 (2013) 986.

[3] M. Czakon and A. Mitov, Comput. Phys. Commun. 185 (2014) 2930.

[4] S. Alioli et al., Eur. Phys. J. C 73 (2013) 2438 [arXiv:1303.6415 [hep-ph]].

[5] G. Aad et al. [ATLAS Collaboration], JHEP 1510 (2015) 121 [arXiv:1507.01769 [hep-ex]].

[6] CMS Collaboration [CMS Collaboration], CMS-PAS-TOP-13-006.

[7] D. L. Rainwater et al., Phys. Rev. D 60, 113004 (1999) Erratum: [Phys. Rev. D 61, 099901 (2000)].

[8] N. Kauer, T. Plehn, D. L. Rainwater and D. Zeppenfeld, Phys. Lett. B 503 (2001) 113.

[9] M. L. Mangano, Eur. Phys. J. C 59 (2009) 373.

[10] M. I. Gresham, I. W. Kim and K. M. Zurek, Phys. Rev. D 84 (2011) 034025.

[11] S. Dittmaier, P. Uwer and S. Weinzierl, Phys. Rev. Lett. 98 (2007) 262002.

[12] S. Dittmaier, P. Uwer and S. Weinzierl, Eur. Phys. J. C 59 (2009) 625.

[13] K. Melnikov and M. Schulze, Nucl. Phys. B 840 (2010) 129.

[14] K. Melnikov, A. Scharf and M. Schulze, Phys. Rev. D 85 (2012) 054002.

[15] A. Denner, S. Dittmaier, S. Kallweit and S. Pozzorini, Phys. Rev. Lett. 106 (2011) 052001.

[16] G. Bevilacqua et al., JHEP 1102 (2011) 083.

[17] A. Denner, S. Dittmaier, S. Kallweit and S. Pozzorini, JHEP 1210 (2012) 110.

[18] R. Frederix, Phys. Rev. Lett. 112 (2014) no.8, 082002.

[19] F. Cascioli, S. Kallweit, P. Maierhöfer and S. Pozzorini, Eur. Phys. J. C 74 (2014) no.3, 2783. 
[20] G. Heinrich, A. Maier, R. Nisius, J. Schlenk and J. Winter, JHEP 1406 (2014) 158.

[21] A. Denner and R. Feger, JHEP 1511 (2015) 209.

[22] G. Bevilacqua, H. B. Hartanto, M. Kraus and M. Worek, Phys. Rev. Lett. 116 (2016) no.5, 052003.

[23] J. Alcaraz Maestre et al., arXiv:1203.6803 [hep-ph].

[24] A. Kardos, C. Papadopoulos and Z. Trocsanyi, Phys. Lett. B 705 (2011) 76.

[25] S. Alioli, S. O. Moch and P. Uwer, JHEP 1201 (2012) 137.

[26] M. Czakon, H. B. Hartanto, M. Kraus and M. Worek, JHEP 1506 (2015) 033.

[27] G. Bevilacqua, M. Czakon, C. G. Papadopoulos, R. Pittau and M. Worek, JHEP 0909 (2009) 109.

[28] G. Bevilacqua, M. Czakon, C. G. Papadopoulos and M. Worek, Phys. Rev. Lett. 104 (2010) 162002.

[29] G. Bevilacqua, M. Czakon, C. G. Papadopoulos and M. Worek, Phys. Rev. D 84 (2011) 114017.

[30] M. Worek, JHEP 1202 (2012) 043.

[31] G. Bevilacqua and M. Worek, JHEP 1207 (2012) 111.

[32] G. Bevilacqua and M. Worek, JHEP 1407 (2014) 135.

[33] A. Kanaki and C. G. Papadopoulos, Comput. Phys. Commun. 132 (2000) 306.

[34] A. Cafarella, C. G. Papadopoulos and M. Worek, Comput. Phys. Commun. 180 (2009) 1941.

[35] A. van Hameren, C. G. Papadopoulos and R. Pittau, JHEP 0909 (2009) 106.

[36] G. Ossola, C. G. Papadopoulos and R. Pittau, Nucl. Phys. B 763 (2007) 147.

[37] G. Ossola, C. G. Papadopoulos and R. Pittau, JHEP 0803 (2008) 042.

[38] A. van Hameren, Comput. Phys. Commun. 182 (2011) 2427.

[39] S. Catani, S. Dittmaier, M. H. Seymour and Z. Trocsanyi, Nucl. Phys. B 627 (2002) 189.

[40] G. Bevilacqua, M. Czakon, M. Kubocz and M. Worek, JHEP 1310 (2013) 204.

[41] M. Czakon, C. G. Papadopoulos and M. Worek, JHEP 0908 (2009) 085.

[42] A. van Hameren, arXiv:1003.4953 [hep-ph].

[43] A. Denner et al., Nucl. Phys. B 724 (2005) 247 Erratum: [Nucl. Phys. B 854 (2012) 504].

[44] [ATLAS and CDF and CMS and D0 Collaborations], arXiv:1403.4427 [hep-ex].

[45] M. Jezabek and J. H. Kühn, Nucl. Phys. B 314 (1989) 1.

[46] A. D. Martin, W. J. Stirling, R. S. Thorne and G. Watt, Eur. Phys. J. C 63 (2009) 189.

[47] M. Cacciari, G. P. Salam and G. Soyez, JHEP 0804 (2008) 063.

[48] S. Chatrchyan et al. [CMS Collaboration], Eur. Phys. J. C 73 (2013) 2494.

[49] G. Aad et al. [ATLAS Collaboration], Eur. Phys. J. C 75 (2015) no.7, 330. 